\title{
Association of Tp-Te/QT Ratio With Ventricular Tachycardia in Patients With Idiopathic Outflow Tract Ventricular Premature Contraction
}

\author{
Arina Prihestri Nugraheni ${ }^{\mathrm{a}, \mathrm{b}}$, Irsad Andi Arso ${ }^{\mathrm{a}}$, Erika Maharani ${ }^{\mathrm{a}, \mathrm{b}}$
}

\begin{abstract}
Background: Idiopathic outflow tract ventricular premature contraction (VPC) can evolve into ventricular tachycardia (VT) via triggered activity mechanism. Transmural dispersion of repolarization (TDR) might play a role in idiopathic outflow tract VT by inducing phase 2 early afterdepolarization (EAD) and serve as the functional substrate for VT. Tp-Te/QT ratio as an arrhythmogenesis index has been reported to be associated with the incidence of ventricular arrhythmia. This study aims to investigate the association between $\mathrm{Tp}-\mathrm{Te} / \mathrm{QT}$ ratio with VT incidence in idiopathic outflow tract VPC.
\end{abstract}

Methods: Observational research with cross sectional design was conducted. VT episodes were retrospectively tracked from electrocardiogram (ECG), treadmill test (TMT), Holter monitor and electrophysiology study data in Sardjito Hospital of patients with idiopathic outflow tract VPC during September to October 2017. Tp-Te/QT was defined as the time from the peak of $\mathrm{T}$ wave to the intersection between the tangent and isoeectric line, divided with QT interval. Tp-Te/QT ratio measurement was performed in leads V4, V5 and V6 by single observer. Tp-Te/QT ratio was categorized into increased $(>0.25)$ and normal $(<0.25)$. Chi-square and logistic regression test were performed.

Results: Out of 46 patients, there were 28 patients who had VT. Increased Tp-Te/QT ratio of lead V4 was found in 11 patients, the increased ratios in leads V5 and V6 were found in 13 patients. The prevalence ratio (PR) of Tp-Te/QT ratio to VT incidence in lead V4 was 2.059 (95\% CI: $1.464-2.895 ; \mathrm{P}=0.007)$, while in leads V5 and V6 was 2.200 (95\% CI: 1.514 - 3.197; P = 0.002). Tp-Te/QT ratios in leads V4, V5 and V6 were not significantly different and equally strong in predicting VT events $(\mathrm{P}<0.001 ; 95 \% \mathrm{CI})$. Adjustment of confounding factor hypertension with multivariate test gave insignifi-

Manuscript submitted May 30, 2018, accepted June 15, 2018

aDepartment of Cardiology and Vascular Medicine, Faculty of Medicine, Universitas Gadjah Mada, Dr. Sardjito General Hospital, Yogyakarta, Indonesia ${ }^{b}$ Corresponding Author: Arina Prihestri Nugraheni, Department of Cardiology and Vascular Medicine, Faculty of Medicine, Universitas Gadjah Mada, Dr. Sardjito General Hospital, Yogyakarta, Indonesia. Email: arina_cdr@yahoo. co.id; Erika Maharani, Department of Cardiology and Vascular Medicine, Faculty of Medicine, Universitas Gadjah Mada, Dr. Sardjito General Hospital, Yogyakarta, Indonesia. Email: erikamaharyo@yahoo.com

doi: https://doi.org/10.14740/cr735w cant results (PR: 1.290; 95\% CI: 0.444 - 3.747).

Conclusions: Increased Tp-Te/QT ratio in idiopathic outflow tract VPC patients was associated with higher prevalence ratio for VT, although this was affected by hypertension. Leads V4, V5 and V6 were equally strong in predicting VT events.

Keywords: Idiopathic outflow tract VPC; VT; Transmural dispersion of repolarization; Tp-Te/QT ratio

\section{Introduction}

Idiopathic ventricular arrhythmia is arrhythmia that can occur in the normal structured heart, without metabolic disturbance nor electrolyte disturbance. Ventricular premature contraction (VPC) and ventricular tachycardia (VT) in the normal structured heart may originate from the left ventricle (idiopathic left ventricular tachycardia/ILVT), ventricular outflow tracts or from other sites such as the mitral annulus, tricuspid annulus, papillary muscle and epicardial perivaskular. The estimated prevalence of VPC in patients with normal heart structure is about $1-4 \%$ of the general population [1], whereas the idiopathic VT is around $10 \%$ of the total patients referred to the arrhythmia center in United States [2] and 20\% in Japan [3].

Triggered activity mechanism is thought to play a role in the progression of idiopathic outflow tract VPC to VT by the occurence of early afterdepolarization (EAD) in the phase 2 repolarization (Fig. 1) which is transmitted transmurally [4, 5]. The functional reentry pathway exists due to the presence of Masonic Midmyocardial Moe (M) cells which during bradycardia or administration of certain drugs, has the ability to prolong its action potential duration over epicardial and endocardial cells [6]. The different action potential duration of epicardial cells, $\mathrm{M}$ cells and endocardial cells results in transmural dispersion of repolarization (TDR) [7].

Several studies found that increased TDR was associated with the occurrence of VT. Some pathological conditions such as long QT syndrome, Brugada syndrome and post-myocardial infarction may affect the normal pattern of repolarization resulting in increased TDR and VT risk [8]. Increased TDR can be detected by prolongation of the Tpeak-Tend (Tp-Te) interval on 12-lead electrocardiogram (ECG). Tp-Te is the time in- 


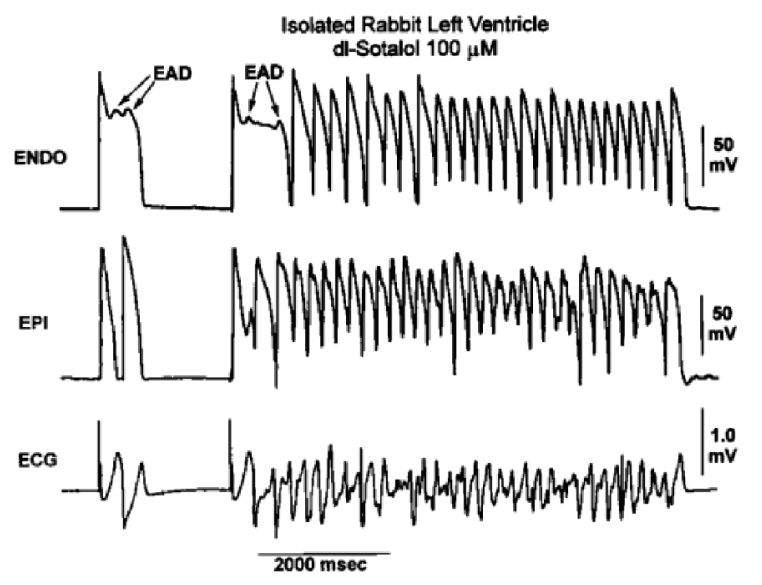

Figure 1. EAD-induced trigger initiated long run of polymorphic VT in arterially perfused rabbit left ventricle pretreated with dl-sotalol 100 $\mathrm{mmol} / \mathrm{L}[5]$.

terval measured from the peak of the $\mathrm{T}$ wave to the end of the $\mathrm{T}$ wave, which coincides with the repolarization time of epicardium cells until the completion of $\mathrm{M}$ cell repolarization [9].

Ratio of Tp-Te/QT has been reported from previous studies could serve as arrhythmogenesis index, reflecting VT susceptibility. The Tp-Te/QT ratio takes into account TDR and compares TDR to the total duration of repolarization time [10]. Increased $\mathrm{Tp}-\mathrm{Te} / \mathrm{QT}$ ratio in studies of Brugada syndrome, long QT syndrome, short QT syndrome and obstructive hypertrophy cardiomyopathy has shown association with ventricular arrhythmia or sudden death $[11,12]$. This study aims to identify the association between Tp-Te/QT ratio with VT incidence in idiopathic outflow tract VPC.

\section{Materials and Methods}

This study was an observational study with cross sectional design, with the subject of idiopathic outflow tract VPC patients in Cardiology Department, Dr. Sardjito Hospital, Yogyakarta. The inclusion criteria were: 1) Idiopathic outflow tract VPC patients; 2) Normal ejection fraction; 3) Patients with age $\geq$ 18 years. Exclusion criteria were: 1) Chronic heart failure; 2) Chronic renal failure; 3) Supraventricular tachycardia (SVT); 4) Primary valvular heart disease; 5) Hypertrophy obstructive cardiomyopathy; 6) Conduction disturbance of His bundles; 7) Pulmonary embolism; 8) Myocarditis; 9) ECG records can not be interpreted e.g. undocumented, atrial fibrillation, flat $\mathrm{T}$ wave, artifact, and multiphasic.

Subject data were retrieved retrospectively in Medical Record Installation and Cardiology Department of Dr. Sardjito Hospital, Yogyakarta, from ECG records, echocardiogram, treadmill test (TMT), coronary computed tomography (CT) scan, Holter monitor and electrophysiology study. The samples data were recorded during February 2013 until July 2017. Events of spontaneous VT were tracked from ECG, TMT or Holter monitor data. ECG was performed with standard 12-lead ECG with speed setting $25 \mathrm{~mm} / \mathrm{s}$ and gain $10 \mathrm{~mm} / \mathrm{mV}$. The TMT was done in Bruce

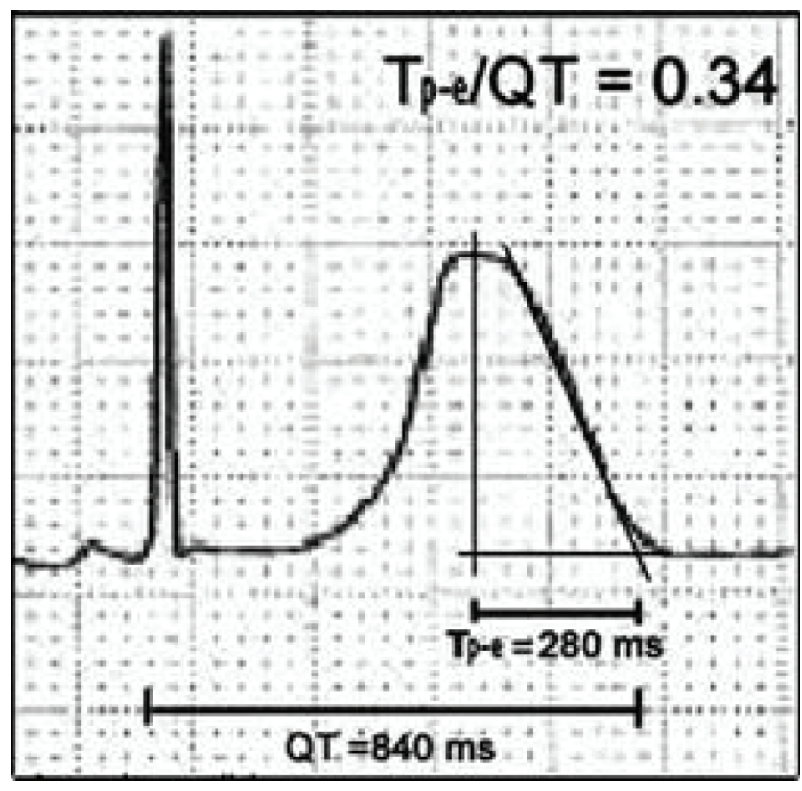

Figure 2. Tp-Te/QT ratio measurement [10].

method using a GE T2100 treadmill manufactured by General Electric Company. Holter monitor recording was performed 24 $\mathrm{h}$ with BTL 08 manufactured by BTL Corporate. VT could also be triggered by programmed electrical stimulation (PES) during electrophysiology studies.

All patient data were recorded in Case Report Form (CRF). Records of ECG was scanned for digital processing. $\mathrm{Tp}-\mathrm{Te} / \mathrm{QT}$ was defined as the time from the peak of T wave to the intersection between the tangent and isoeectric line, divided with QT interval. Measurement of Tp-Te/QT ratio in leads V4, V5 and V6 was done in tangent method (Fig. 2) [10]. Measurements of Tp-Te and QT values were performed using ImageJ program. A single-blind obeserver performed the ECG measurement and previously tested with Kappa. The kappa value for intra and inter-observer variability were 0.826 and 0.783 respectively.

Statistical analysis was performed using SPSS for Windows software version 22.0. A comparative analysis was done in independent $t$-test to compare $\mathrm{Tp}$-Te/QT ratio between groups with VT event group and those without VT events. Ratio of Tp-Te/QT then was classified into normal $(<0.25)$ and increased $(>0.25)$ groups. The hypothesis test was performed in Chi-square test.

\section{Ethics}

Research was conducted after receiving a permission from Medical Faculty of Universitas Gadjah Mada/Dr. Sardjito General Hospital Ethical Commitee.

\section{Results}

Among 64 idiopathic VPC patients, 46 patients were recruited 


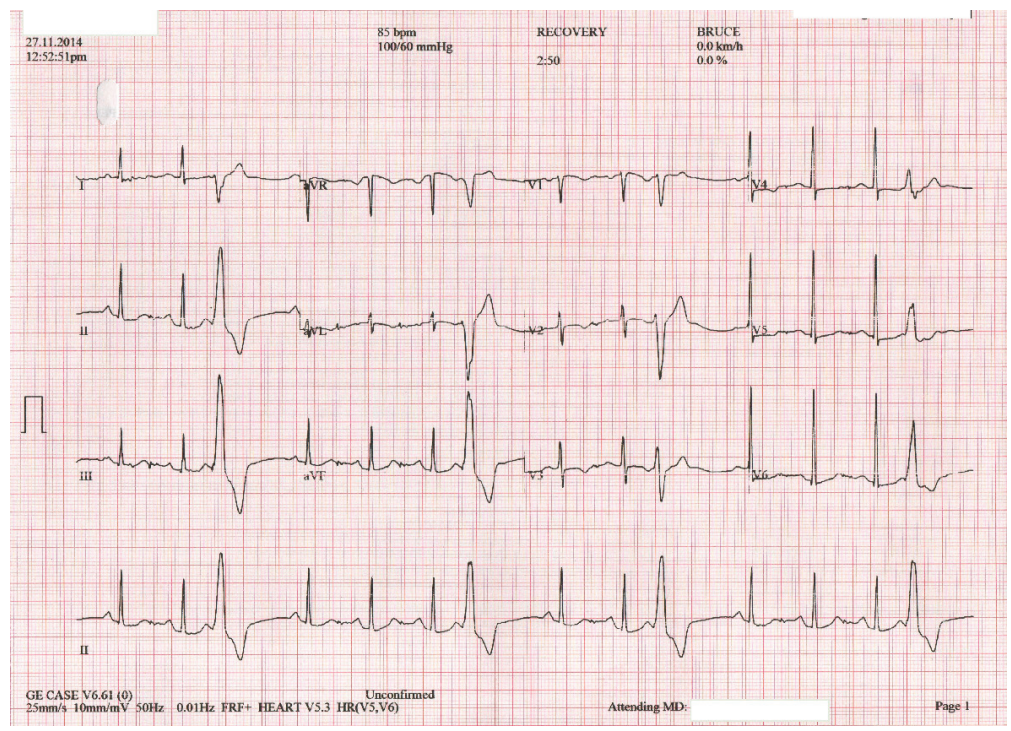

Figure 3. ECG from idiopathic outflow tract VPC patient.

based on the inclusion and exclusion criteria. ECG samples of VPC and VT patients could be seen in Figure 3 and 4. There were 11 patients with incomplete data and seven patients which were loss of follow-up. There were 32 (69.6\%) female patients and $14(30.4 \%)$ male patients. Mean age in all subjects was $44.59 \pm 10.939$ years. The coexisting disease in the study subjects were hypertension in 15 patients $(32.6 \%)$ with left ventricular hypertrophy in five patients $(10.9 \%)$, while diabetes mellitus (DM) was present in four patients $(8.7 \%)$ and no SVT was observed. All patients in the study sample were normokinetic and had normal ejection fraction with mean $63.6 \pm 6.099 \%$. The basic characteristics of the patient can be seen from Table 1.

Clinical complaints experienced were mostly palpitation in 29 patients $(63 \%)$ while unstable VT with hemodynamic disorders occured in two (4.3\%) cases. Some ECGs were recorded at the time when patients were taking antiarrhythmic drugs i.e. bisoprolol in five $(10.8 \%)$ patients, metoprolol in one $(2.1 \%)$ patient, and verapamil also in one $(2.1 \%)$ patient. The use of antihypertensive drugs was found mostly from angiotensin converting enzyme (ACE) inhibitor and angiotensin receptor blocker (ARB) class.

Based on VT incidence, 28 VT patients and 18 non-VT patients (Table 2) were found. Hypertension was more prevalent in the group with the VT incidence $(\mathrm{P}=0.003 ; 95 \% \mathrm{CI})$. The incidence of spontaneous VT occured in 21 patients $(75 \%)$ and the rest experienced VT induced during electrophysiological studies. The most common arrhythmia source was originated from the septal right ventricular outflow tract (RVOT) with a percentage of $50 \%$ (nine patients) in the non-VT group and

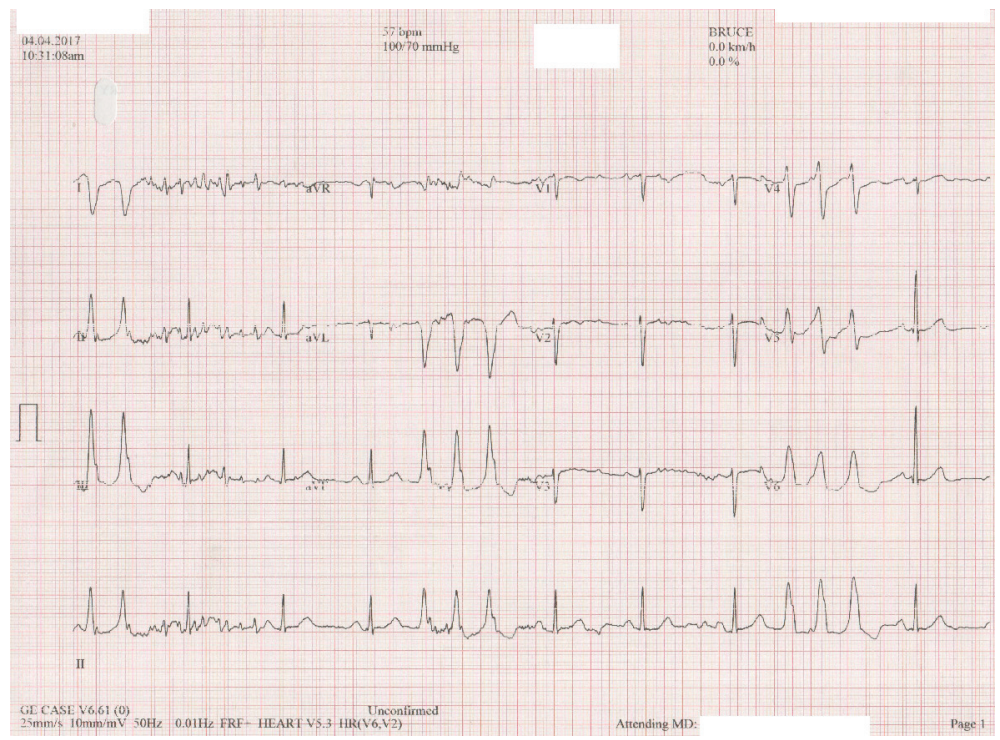

Figure 4. ECG from idiopathic outflow tract VT patient. 
Table 1. Basic Characteristics of the Idiopathic Outflow Tract VPC Subjects $(N=46)$

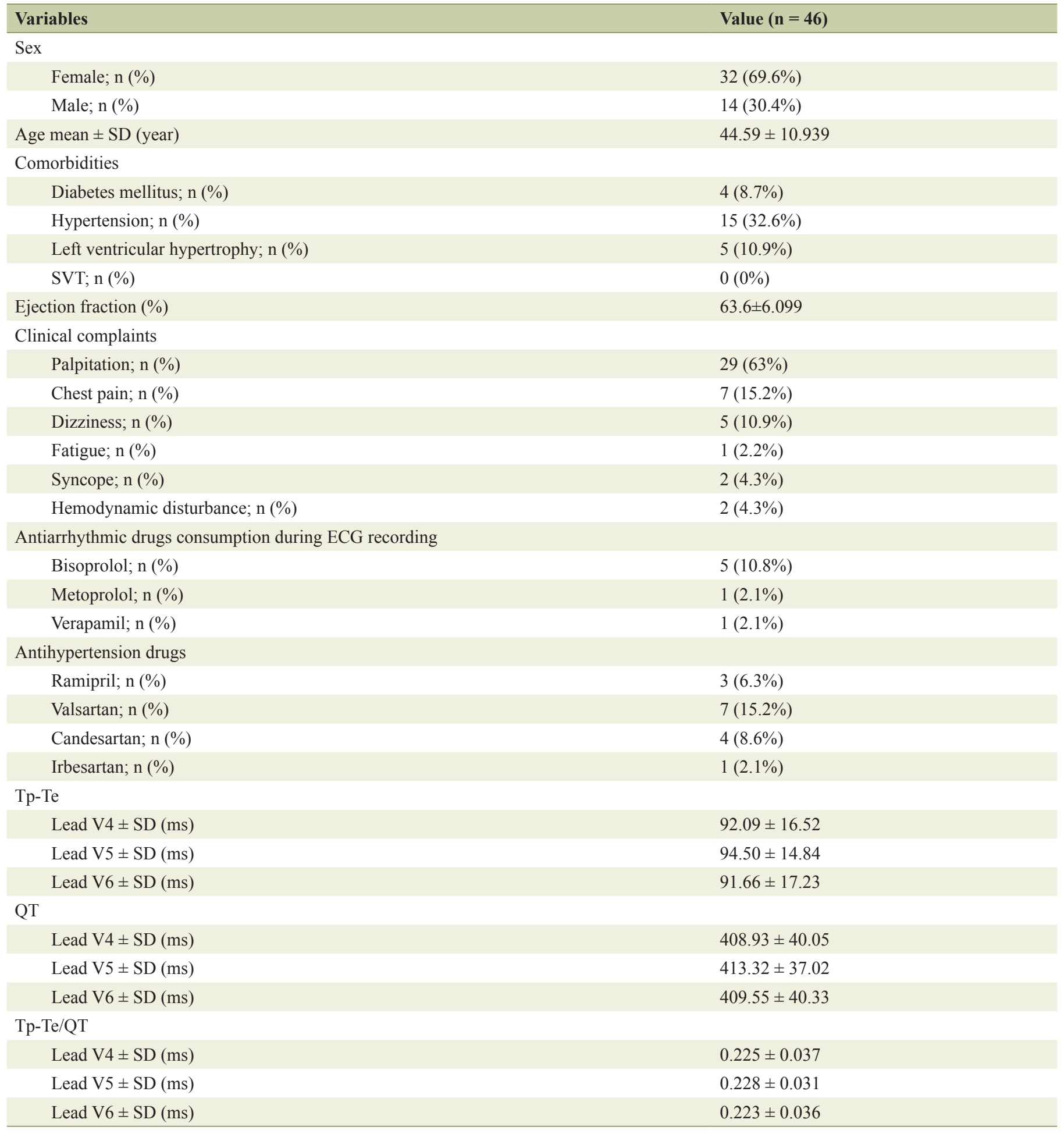

ECG: electrocardiogram; SD: standard deviation; SVT: supraventricular tachycardia.

$25 \%$ (seven patients) in the VT group, followed by anteroseptal RVOT, posteroseptal RVOT and free wall. The LVOT (left ventricular outflow tract) area as the source of arrhythmia activity was obtained in only one subject.
The ECG markers for QT interval, Tp-Te interval and TpTe/QT ratio differed between groups of VT patients compared to non-VT groups. Longer QT interval was measured in the VT group $(\mathrm{P}=0.023$ for lead $\mathrm{V} 4 ; \mathrm{P}=0.039$ for lead $\mathrm{V} 5 ; \mathrm{P}=0.022$ 
Table 2. Characteristics of Research Subjects Based on VT Incidence ( $N=46)$

\begin{tabular}{|c|c|c|c|}
\hline Variables & Without VT $(n=18)$ & With VT $(n=28)$ & $\mathbf{P}$ \\
\hline \multicolumn{4}{|l|}{ Sex } \\
\hline Female; n (\%) & $11(69.6 \%)$ & $21(75 \%)$ & 0.502 \\
\hline Male; n (\%) & $7(30.4 \%)$ & $7(25 \%)$ & \\
\hline Age, mean \pm s.d. (year) & $40.5 \pm 11.284$ & $47.21 \pm 10.049$ & 0.41 \\
\hline \multicolumn{4}{|l|}{ Comorbidities } \\
\hline Diabetes mellitus; $\mathrm{n}(\%)$ & $1(5.8 \%)$ & $3(10.7 \%)$ & 0.944 \\
\hline Left ventricular hypertrophy; n (\%) & $2(11.6 \%)$ & $4(14.3 \%)$ & 0.634 \\
\hline SVT; n $(\%)$ & $0(0 \%)$ & $0(0 \%)$ & - \\
\hline Ejection fraction & $63.28 \pm 2.824$ & $63.68 \pm 7.484$ & 0.829 \\
\hline \multicolumn{4}{|l|}{ Drug consumption during ECG } \\
\hline Verapamil; n (\%) & $0(0 \%)$ & $1(3.5 \%)$ & 1 \\
\hline Ramipril & $1(5.5 \%)$ & $2(7.1 \%)$ & 1 \\
\hline Valsartan & $1(5.5 \%)$ & $6(21.4 \%)$ & 0.297 \\
\hline Candesartan & $0(0 \%)$ & $4(14.3 \%)$ & 0.891 \\
\hline Irbesartan & $0(0 \%)$ & $1(3.5 \%)$ & 1 \\
\hline \multicolumn{4}{|l|}{ VT incidence } \\
\hline Spontaneous VT & & $21(75 \%)$ & \\
\hline Induced VT & & $7(25 \%)$ & \\
\hline \multicolumn{4}{|l|}{ Types of VT } \\
\hline Nonsustained VT & - & $16(57.1 \%)$ & \\
\hline Sustained VT & - & $10(35.7 \%)$ & \\
\hline Free wall RVOT & $2(11.1 \%)$ & $1(3.6 \%)$ & 0.552 \\
\hline LVOT & $1(5.6 \%)$ & $0(0 \%)$ & 0.391 \\
\hline \multicolumn{4}{|l|}{$\mathrm{Tp}-\mathrm{Te}$} \\
\hline Lead V4 \pm SD (ms) & $75.83 \pm 11.86$ & $102.54 \pm 8.76$ & $<0.001 *$ \\
\hline Lead V5 \pm SD (ms) & $80.50 \pm 8.49$ & $103.50 \pm 10.34$ & $<0.001^{*}$ \\
\hline Lead V6 $\pm \mathrm{SD}(\mathrm{ms})$ & $74.76 \pm 8.56$ & $102.53 \pm 11.67$ & $<0.001 *$ \\
\hline \multicolumn{4}{|l|}{ QT } \\
\hline Lead V4 \pm SD (ms) & $392.37 \pm 39.33$ & $419.58 \pm 37.40$ & $0.023^{*}$ \\
\hline Lead V $5 \pm$ SD (ms) & $399.37 \pm 35.96$ & $422.30 \pm 35.44$ & $0.039 *$ \\
\hline Lead V6 \pm SD (ms) & $392.75 \pm 33.99$ & $420.35 \pm 40.91$ & $0.022 *$ \\
\hline \multicolumn{4}{|l|}{ Ratio Tp-Te/QT } \\
\hline Sandapan V4 \pm SD (ms) & $0.194 \pm 0.033$ & $0.245 \pm 0.025$ & $<0.001 *$ \\
\hline Sandapan V5 \pm SD (ms) & $0.202 \pm 0.021$ & $0.245 \pm 0.024$ & $<0.001 *$ \\
\hline Sandapan V6 \pm SD (ms) & $0.191 \pm 0.022$ & $0.245 \pm 0.028$ & $<0.001^{*}$ \\
\hline
\end{tabular}

VT: ventricular tachycardia; ECG: electrocardiogram; RVOT: right ventricular outflow tract; LVOT: left ventricular outflow tract; SVT: supraventricular tachycardia; SD: standard deviation. ${ }^{*} \mathrm{P}<0.05 ; 95 \% \mathrm{Cl}$. 


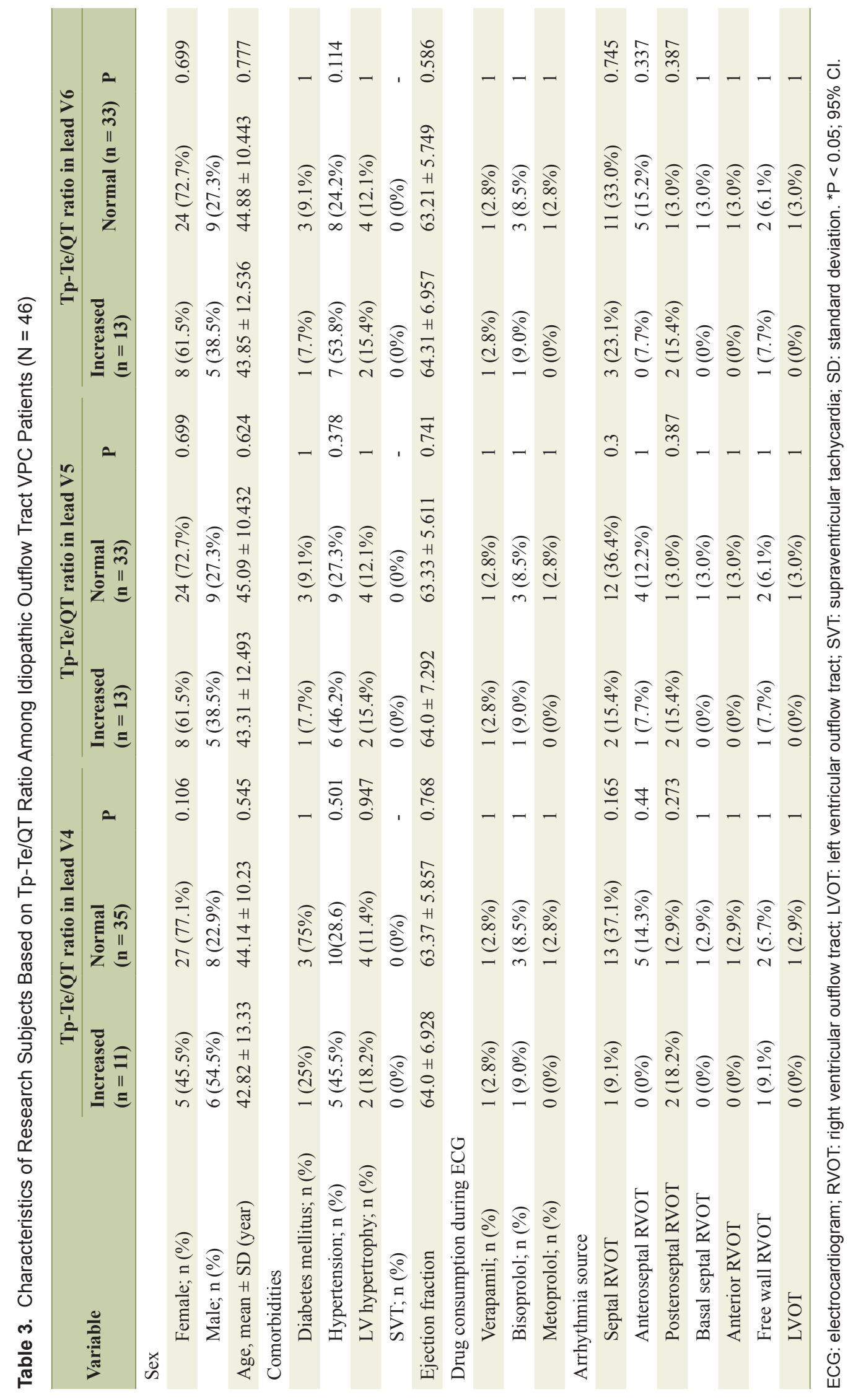


Table 4. Prevalence Ratio of Tp-Te/QT Ratio to VT Incidence Among Idiopathic Outflow Tract VPC Patients

\begin{tabular}{llll}
\hline Variable & PR & $\mathbf{9 5 \%}$ CI & P \\
\hline $\begin{array}{c}\text { Tp-Te/QT ratio } \\
\text { Lead V4 }\end{array}$ & 2.059 & $1.464-2.895$ & $0.007^{*}$ \\
Lead V5 & 2.200 & $1.514-3.197$ & $0.002 *$ \\
Lead V6 & 2.200 & $1.514-3.197$ & $0.002 *$ \\
\hline
\end{tabular}

Tp-Te: Tpeak-Tend interval; PR: prevalence ratio. ${ }^{*} \mathrm{P}<0.05 ; 95 \% \mathrm{Cl}$.

for lead V6; 95\% CI) and also for Tp-Te interval $(\mathrm{P}<0.001$ for lead V4; $\mathrm{P}<0.001$ for lead V5; $\mathrm{P}<0.001$ for lead V6; $95 \%$ $\mathrm{CI})$. In this study, mean of $\mathrm{Tp}-\mathrm{Te} / \mathrm{QT}$ ratio measured in those three leads were all found to be longer in the VT group $(\mathrm{P} \leq$ $0.001 ; 95 \%$ CI $)$ in comparison with the group without VT. The lead V4 Tp-Te/QT ratio mean value was around $0.245 \pm 0.025$ vs. $0.194 \pm 0.033 ; 0.245 \pm 0.024$ vs. $0.202 \pm 0.021$ in lead V5; and $0.245 \pm 0.028$ vs. $0.191 \pm 0.022$ in lead V6.

In Table 3 the subjects then were further categorized into normal $(<0.25)$ and increased $(>0.25)$ Tp-Te/QT ratio measured in leads V4, V5 and V6. There were 27 patients with normal and 11 patients with increased Tp-Te/QT ratio in lead V4, while in leads V5 and V6 were the same with 33 normal patients and 13 patients with increased Tp-Te/QT ratio. All groups were dominated by female, except in the group with increased Tp-Te/QT ratio in lead V4 (45.5\% female) though it was not significantly different $(\mathrm{P}=0.106 ; 95 \% \mathrm{CI})$. Mean of age were all around 40 years, mean ejection fraction (EF) was around $60 \%$, comparable comorbidities and drug consumptions during ECG $(\mathrm{P}=1.000 ; 95 \% \mathrm{CI})$.

From Chi-square analysis (Table 4), significant risk for VT incidence $(\mathrm{P}<0.005 ; 95 \% \mathrm{CI})$ was found for patients with increased $\mathrm{Tp}-\mathrm{Te} / \mathrm{QT}$ ratio with prevalence ratio (PR) of 2.059 in lead V4 measurement and PR of 2.200 for measurements either in V5 or V6. The Tp-Te/QT ratio either was performed in lead $\mathrm{V} 4, \mathrm{~V} 5$ or $\mathrm{V} 6$ each gave very similar values $(\mathrm{P}<0.001 ; 95 \%$ $\mathrm{CI}$ ) in one-way ANOVA (Table 5). Logistic regression test was done (Table 6) on variables that could potentially serve as confounding factors and lead V6 was chosen considering higher PR compared to lead V4 and was the lead used in the reference study by Gupta et al (2008) [10]. Taking into account hypertension as the confounding factor, Tp-Te/QT ratio in lead V6 gave insignificant result (PR 1.290; $\mathrm{P}=0.639 ; 0.444$ - 3.747).

Table 5. Comparison of Tp-Te/QT Ratio Measurements in Leads V4, V5 and V6

\begin{tabular}{llll}
\hline Lead & VT incidence & Mean Tp-Te/QT \pm SD $(\mathbf{m s})$ & $\mathbf{P}$ \\
\hline V4 & Yes & $0.245 \pm 0.025$ & $<0.001^{*}$ \\
& No & $0.194 \pm 0.033$ & $<0.001^{*}$ \\
V5 & Yes & $0.245 \pm 0.024$ & $<0.001^{*}$ \\
& No & $0.202 \pm 0.021$ & $<0.001^{*}$ \\
V6 & Yes & $0.245 \pm 0.028$ & $<0.001^{*}$ \\
& No & $0.191 \pm 0.022$ & $<0.001^{*}$ \\
\hline
\end{tabular}

Tp-Te: Tpeak-Tend interval; SD: standard deviation. ${ }^{*} \mathrm{P}<0.05 ; 95 \% \mathrm{Cl}$.
Table 6. Multivariate Analysis of VT Incidence in Idiopathic Outflow Tract VPC Patients

\begin{tabular}{llll}
\hline Variable & Exp (B) & $\mathbf{9 5 \%}$ CI & P \\
\hline Hypertension & 0.685 & $0.230-2.043$ & 0.497 \\
Tp-Te/QT ratio (lead V6) & 1.29 & $0.444-3.747$ & 0.639 \\
\hline
\end{tabular}

Tp-Te: Tpeak-Tend interval; Exp (B): exponential beta. ${ }^{*} \mathrm{P}<0.05 ; 95 \%$ Cl.

\section{Discussions}

In this study, subjects were dominated by female (69.6\%) with mean age was $44.59 \pm 10.939$ years, the youngest subject was 22 years old, and the oldest one was 62 years old. Ventricular arrhythmia source was mostly coming from RVOT (96.8\%) and only one patients had LVOT VPC. This findings matched with previous studies [2-4] stating that idiopathic RVOT arrhythmia happened two times more often in female and mostly occured in the third to fifth decade of life with mean presentation in the age of early 40s. Diabetes mellitus and hypertension were found to be the comorbidities in these patients, with more hypertensive patients were found in the VT group. Increased left ventricular mass in the hypertensive patients was thought could contribute to longer Tp-Te interval, though left ventricular hypertrophy was found similar in both groups (Table 2).

Increased dispersion of transmural repolarization in this study was characterized by the prolongation of Tp-Te interval. The Tp-Te interval in the leads V4, V5 or V6 from the bivariate test was found to be longer $(\mathrm{P}<0.001 ; 95 \% \mathrm{CI})$ in idiopathic outflow tract VPC experiencing VT either spontaneously or induced by electrophysiological studies (Table 2). Several previous clinical studies have found similar results which was the association between transmural repolarization dispersion using Tp-Te parameters with ventricular arrhythmias. During ventricular pacing, Tp-Te was found to be longer in VT-induceable patients compared with the non VT-induceable patients [13]. Patients with sustained VT and VF were also reported to have mean 12-lead Tp-Te values significantly longer compared to normal subjects [14]. In another study there was prolongation of lead V4 Tp-Te interval in patients experiencing VT-induced PES [15], while in another study it was found that Tp-Te was significantly longer in the idiopathic outflow tract VPC group who developed monomorphic VT [16].

In this study Tp-Te/QT ratio of leadsV4, V5 and V6 were all significantly longer in the group of patients with VT compared with those without VT incidence $(\mathrm{P}<0.001 ; 95 \% \mathrm{CI})$ in bivariate analysis. Based on references from studies by Gupta et al [10], the normal range values for $\mathrm{Tp}-\mathrm{Te} / \mathrm{QT}$ ratio was from $0.15-0.25$ with median 0.21 and mean $0.21 \pm 0.003$. The research subjects in this study were then divided into two groups: those with normal Tp-Te/QT ratio $(<0.25)$ and increased $(>0.25)$ based on measurements done in leads V4, V5 and V6. No significantly different baseline characteristics of patients were found between the two groups (Table 3 ).

Measurement of Tp-Te/QT ratio was performed on leads V4, V5 and V6 with no significant difference $(\mathrm{P}<0.001 ; 95 \%$ CI). One-way ANOVA tests showed that V4, V5 and V6 can be 
used each equally strong in distinguishing idiopathic outflow tract VPC patients experiencing VT events and those without VT events (Table 5). In this study increased Tp-Te/QT ratio in leads V4, V5 or V6 was found to have elevated risk for VT incidence. Increased Tp-Te/QT ratio in lead V4 has 2.059 times the risk to experience VT incidence compared with normal TpTe/QT, while the VT risk was slightly higher as 2.200 times, if increased $\mathrm{Tp}-\mathrm{Te} / \mathrm{QT}$ ratio was found in leads V5 and V6. The measurements in leads V4, V5 and V6 gave similar Tp-Te and $\mathrm{Tp}-\mathrm{Te} / \mathrm{QT}$ values, as evidenced with significant bivariate study analysis (Table 2) and statistically significant PR test (Table 4). Unfortunately, this significant elevated risk was showed to be insignificant after considering hypertension as confounding factor (table 6). In this study Tp-Te/QT ratio has not been able to serve as an independent predictor for VT incidence in idiopathic outflow tract VPC patients, due to the confounding effect of hypertension. Patients with increased Tp-Te/QT ratio who possesed hypertension as well, had elevated risk for VT but this cannot be applied for patients who were not hypertensive.

In the previous studies, it was found that $\mathrm{Tp}-\mathrm{Te} / \mathrm{QT}$ ratio could serve as an index of arithmogenesis to predict ventricular arrhythmia in certain susceptible populations. Ratio of TpTe/QT in long QT syndrome patients may act as a predictor of ventricular arrhythmia [11]. In a study of obstructive cardiomyopathy patients who experienced sudden cardiac death, an increased Tp-Te /QT ratio was observed [12]. Increased Tp-Te/ QT ratio was also found in the study of VT-induced high risk Brugada syndrome patients $[17,18]$, while in other idiopathic VPC study Tp-Te/QT ratio was shorter after successful radiofrequency ablation therapy $(\mathrm{P}=0.023 ; 95 \% \mathrm{CI})$ [19]. The application of this Tp-Te/QT ratio study was hopefully, and could be used in the peripheral or remote areas which are limited in resources, to distinguish idiopathic outflow tract VPC patients who had elevated risk for developing VT and could be managed further or referred to other cardiac centres.

\section{Limitations}

Study by Gupta et al (2008) [10] used references from normal population samples while in this study, normal patients without arrhythmia disorder as control group were not recruited. It is possible that there are actually variations in normal value in Indonesian population because: 1) Samples in reference studies were western populations; 2) The sample population in this study basically have an arrhythmia disorder in the form of an idiopathic outflow tract VPC so the range of values may not be equal with a completely normal population.

\section{Conclusions}

Increased Tp-Te/QT ratio in idiopathic outflow tract VPC patients is associated with higher prevalence ratio for VT incidence, but this association is not independent due to the influence of hypertension. Measurements of Tp-Te/QT ratio in leads V4, V5 and V6 can be used equally strong in predicting VT incidence.

\section{Acknowledgments}

We would like to thank all of the Cardiology staffs in Sardjito General Hospital.

\section{Conflict of Interest}

None.

\section{Funding Support}

None.

\section{Abbreviations}

ACE: angiotensin converting enzyme; ARB: angiotensin receptor blocker; $\mathrm{CI}$ : confidence interval; CRF: case report file; DM: diabetes mellitus; EAD: early after depolarization; ECG: electrocardiogram; ILVT: idiopathic left ventricular tachycardia; LVOT: left ventricular outflow tract; M cell: Masonic midmyocardial Moe cells; QTd: QT dispersion; PR: prevalence ratio; RVOT: right ventricular outflow tract; PES: programmed electrical stimulation; SD: standard deviation; SVT: supraventricular tachycardia; TdP: torsade de pointes; TDR: transmural dispersion of repolarisation; TMT: treadmill test; Tp-Te: Tpeak-Tend; VPC: ventricular premature contraction; VT: ventricular tachycardia

\section{References}

1. Kennedy HL, Whitlock JA, Sprague MK, Kennedy LJ, Buckingham TA, Goldberg RJ. Long-term follow-up of asymptomatic healthy subjects with frequent and complex ventricular ectopy. N Engl J Med. 1985;312(4):193-197.

2. Hoffmayer KS, Gerstenfeld EP. Diagnosis and management of idiopathic ventricular tachycardia. Curr Probl Cardiol. 2013;38(4):131-158.

3. Badhwar N, Scheinman MM. Idiopathic ventricular tachycardia: Diagnosis and management. Curr Probl Cardiol. 2007;32(1):7-43.

4. Altemose GT, Buxton AE. Idiopathic ventricular tachycardia. Annu Rev Med. 1999;50:159-177.

5. Yan GX, Wu Y, Liu T, Wang J, Marinchak RA, Kowey PR. Phase 2 early afterdepolarization as a trigger of polymorphic ventricular tachycardia in acquired longQT syndrome : direct evidence from intracellular recordings in the intact left ventricular wall. Circulation. 2001;103(23):2851-2856.

6. Antzelevitch C, Sicouri S, Litovsky SH, Lukas A, Krishnan SC, Di Diego JM, Gintant GA, et al. Heterogeneity within the ventricular wall. Electrophysiology and pharmacology of epicardial, endocardial, and $\mathrm{M}$ cells. Circ Res. 1991;69(6):1427-1449.

7. Killeen MJ, Sabir IN, Grace AA, Huang CL. Disper- 
sions of repolarization and ventricular arrhythmogenesis: lessons from animal models. Prog Biophys Mol Biol. 2008;98(2-3):219-229.

8. Antzelevitch C, Shimizu W, Yan GX, Sicouri S. Cellular basis for QT dispersion. J Electrocardiol. 1998;30(Suppl):168-175.

9. Haarmark C, Hansen PR, Vedel-Larsen E, Pedersen SH, Graff C, Andersen MP, Toft E, et al. The prognostic value of the Tpeak-Tend interval in patients undergoing primary percutaneous coronary intervention for ST-segment elevation myocardial infarction. J Electrocardiol. 2009;42(6):555-560.

10. Gupta P, Patel C, Patel H, Narayanaswamy S, Malhotra B, Green JT, Yan GX. T(p-e)/QT ratio as an index of arrhythmogenesis. J Electrocardiol. 2008;41(6):567-574.

11. Yamaguchi M, Shimizu M, Ino H, Terai H, Uchiyama K, Oe K, Mabuchi T, et al. T wave peak-to-end interval and QT dispersion in acquired long QT syndrome: a new index for arrhythmogenicity. Clin Sci (Lond). 2003;105(6):671676.

12. Shimizu M, Ino $H$, Okeie $K$, Yamaguchi $M$, Nagata $M$, Hayashi K, Itoh H, et al. T-peak to T-end interval may be a better predictor of high-risk patients with hypertrophic cardiomyopathy associated with a cardiac troponin I mutation than QT dispersion. Clin Cardiol. 2002;25(7):335339.

13. Wolk R, Stec S, Kulakowski P. Extrasystolic beats affect transmural electrical dispersion during programmed elec- trical stimulation. Eur J Clin Invest. 2001;31(4):293-301.

14. Kuroda N, Ohnishi Y, Okajima K, Ishida A, Kitamura $\mathrm{H}$, Yoshida A, et al. Examination of the dispersion of the latter half of $\mathrm{T}$ wave in patients with fatal ventricular arrhythmia. Japanese Journal of Electrocardiology. 2002;22(4):256-265.

15. Watanabe N, Kobayashi Y, Tanno K, Miyoshi F, Asano T, Kawamura M, Mikami Y, et al. Transmural dispersion of repolarization and ventricular tachyarrhythmias. J Electrocardiol. 2004;37(3):191-200.

16. Yuanjun S, Xiao X, Yin X, Chang D, Dong Y, Gao L, et al. Electrocardiogram predictors of transition from idiopathic outflow tract ventricular premature to monomorphic tachycardia. Journal of the American College of Cardiology. 2016;68(16):C113.

17. Zumhagen S, Zeidler EM, Stallmeyer B, Ernsting M, Eckardt L, Schulze-Bahr E. Tpeak-Tend interval and Tpeak-Tend/QT ratio in patients with Brugada syndrome. Europace. 2016;18(12):1866-1872.

18. Letsas KP, Weber R, Astheimer K, Kalusche D, Arentz T. Tpeak-Tend interval and Tpeak-Tend/QT ratio as markers of ventricular tachycardia inducibility in subjects with Brugada ECG phenotype. Europace. 2010;12(2):158159.

19. Yayla C, Ozcan F, Aras D, Turak O, Ozeke O, Cay S, Topaloglu S. Tp-e interval and Tp-e/QT ratio before and after catheter ablation in patients with premature ventricular complexes. Biomark Med. 2017;11(4):339-346. 